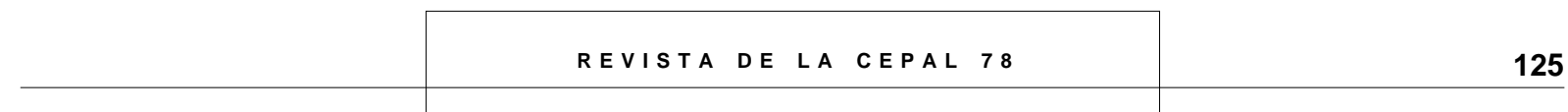

\title{
Equidad en la
}

\section{atención médica del adulto mayor en Chile: papel de la previsión}

\section{Steven P. Wallace}

Profesor de la Escuela de Salud Pública

y Director Adjunto del Centro de Investigaciones de Políticas de Salud de la Universidad de California Los Ángeles (UCLA) Becario Fulbright destacado en la CEPAL, 2000

Swallace@ucla.edu
Los gobiernos cumplen una labor fundamental en la organización y financiamiento de los servicios de salud, y sus intervenciones para promover la equidad en la atención sanitaria contribuyen a su legitimidad. El sistema de seguro de salud y de prestaciones de salud que se aplica en Chile ha originado reservas en lo que concierne a la equidad del financiamiento de los servicios. Aquí se amplía el análisis para examinar la equidad en el acceso a estos servicios, haciendo hincapié en sus principales usuarios, esto es, las personas de 65 años o más. En Chile, la encuesta CASEN de 1998 mostró serias desigualdades tanto entre el sistema público y el privado de salud como dentro de uno y otro. La persistencia de desigualdades en el sistema público de salud indica que, aunque mejoren la equidad y eficiencia financieras, para reducir la inequidad en el acceso a los servicios será preciso prestar mayor atención a los obstáculos que dificultan la obtención de cuidado médico. 


\section{I}

\section{Antecedentes}

La intervención del Estado en los servicios de salud desempeña un importante papel, tanto material como simbólico, en la sociedad (Esping-Andersen, 1994). Por una parte, los gobiernos promueven y regulan los servicios médicos para mantener sanas a las personas y tratar a los enfermos, actividades que en la mayoría de los países absorben parte considerable del producto interno bruto (PIB), y por otra, tienen además una importante función simbólica, pues brindan un beneficio manifiesto a la población en general. En este sentido, la participación del Estado en el sistema de salud contribuye a legitimarlo ante la sociedad. Para cumplir con esta función legitimadora, el sistema que proporcione no sólo debe ser eficaz sino también equitativo. Como las personas de la tercera edad son las mayores usuarias de los servicios de salud y además suelen constituir uno de los grupos sociales con más "legítimo" derecho a obtener prestaciones públicas, la equidad de la política pública en materia de atención médica del adulto mayor permite formarse una impresión bastante acertada acerca de la legitimidad del sistema. Los gobiernos de América Latina están atribuyendo creciente importancia a la atención de salud de las personas de la tercera edad, debido a que varios países (Cuba, Uruguay, Argentina y Chile) se encuentran en una etapa avanzada de transición demográfica, en que más del $10 \%$ de la población tiene 60 años o más y tanto las tasas de natalidad como de mortalidad son bajas (Villa y Rivadeneira, 2000). Esta situación va acompañada de una transición epidemiológica, en virtud de la cual la carga principal de la enfermedad se ha desplazado desde las enfermedades agudas a las crónicas, que se concentran en la población de mayor edad (OPS, 1998). En Chile, 29\% de las familias que viven en zonas urbanas y $37 \%$ de los hogares rurales incluyen a personas mayores (CEPAL, 2001), lo que indica que una parte significativa de la población se ve directamente afectada por los servicios de salud que reciben los más ancianos.

Chile fue uno de los primeros países de América Latina en aplicar "políticas de ajuste estructural" en los años setenta y ochenta. Adhiriendo a principios neoliberales, el gobierno militar adoptó políticas nuevas encaminadas a reducir la función del Estado en la economía y la sociedad. Aunque la privatización y la capitalización individual del sistema público de pen- siones y del sistema de seguro de salud fueron concebidas para hacer frente a la fragmentación y la falta de financiamiento, el Estado ha seguido desempeñando un papel importante en estos ámbitos, en la medida en que subvenciona y regula ambos sistemas (Morley, Machado y Pettinato, 1999; Arenas de Mesa, 2000). En el campo de la salud, la mayoría de los trabajadores de clase media (de mejor situación económica) del sector formal se trasladaron desde el plan de salud público, constituido por el Servicio Médico Nacional de Empleados (sERMENA), a empresas de seguros de salud privadas llamadas instituciones de salud previsional (Isapres). Otros trabajadores asalariados optaron por afiliarse al Fondo Nacional de Salud (FONASA), nuevo sistema administrado y subvencionado por el Estado, y, finalmente, los miembros de las Fuerzas Armadas conservaron su propio sistema de salud. Desde el punto de vista de la organización, en el sector público y en el privado las prestaciones médicas quedaron separadas del seguro propiamente tal, lo que se tradujo en un sistema pluralista en lo referente a estos dos aspectos (Borzutsky, 1999). En los años noventa, el sector público procuró, con éxito moderado pero desigual, separar aún más sus funciones como regulador, proveedor y comprador de atención médica (Sojo, 1999).

En Chile, el seguro médico se "compra" mediante una cotización uniforme obligatoria, equivalente a $7 \%$ de la remuneración del trabajador (sin aportes del empleador). Esta "prima" puede aplicarse al seguro del sistema público (FONASA), al de las numerosas Isapres o al sistema de las Fuerzas Armadas. ${ }^{1}$ El seguro estatal y el privado se centran en hospitalización, consultas médicas y exámenes de laboratorio o rayos $\mathrm{X}$, con una variedad de copagos y aranceles médicos (que en la práctica constituyen límites al monto de los pagos de las aseguradoras y a las prestaciones). Las compañías aseguradoras (y también el Estado) celebran convenios con algunos proveedores privados de atención médica en virtud de los cuales éstos aceptan como pago

\footnotetext{
${ }^{1}$ El personal de las Fuerzas Armadas aporta el 6\% de su sueldo, mientras que el Estado paga el 1\% restante; hasta 1998, sus pensionados sólo eran atendidos dentro del sistema de seguro de salud de las Fuerzas Armadas, pero ahora pueden optar por utilizar sus cotizaciones para ser atendidos por el FONASA o una Isapre.
} 
total la suma establecida en un arancel fijo. Los honorarios que cobran otros proveedores privados pueden exceder, a veces sustancialmente, el arancel máximo que pagan las aseguradoras. Los hospitales y consultorios estatales siempre aceptan los planes de pago del sistema público y, además, reciben a pacientes afiliados a seguros privados.

El seguro estatal es un sistema de cuatro niveles basado en el ingreso del beneficiario. Los indigentes, esto es, las personas que no perciben ingresos, pertenecen al nivel A del FONASA -que no exige copago- y son atendidas exclusivamente en los consultorios y hospitales públicos. Las personas cuyos ingresos se aproximan al salario mínimo y que cotizan el 7\% pueden pertenecer al nivel FONASA c, que tampoco contempla un copago por el uso de los servicios de salud públicos, pero, junto con los afiliados al FONASA C y D, tienen acceso al sistema de "libre elección", en virtud del cual pueden recibir atención médica en el sector privado (mediante un copago de $50 \%$ o más, de acuerdo con un arancel preestablecido). Las personas con mayores ingresos quedan adscritas al FONASA C o el D, con un copago de $10 \%$ y $20 \%$, respectivamente, cuando utilizan el sistema público, sobre la base de aranceles que generalmente son inferiores a los que aplican los médicos que ejercen libremente la profesión (Banco Mundial, 1995). El fonAsA A у в son subvencionados por el fisco, porque su costo es superior a lo recaudado con el pago de la prima, mientras que el FONASA C y D redistribuye algunos recursos, porque, en total, sus costos son inferiores a las cotizaciones recaudadas (Bitrán y otros, 1996). El sistema de seguro de salud de las Fuerzas Armadas también contempla un plan de libre elección, cuyos límites son similares a los de los planes afines del FONASA.

En el mercado privado de seguros de salud, las personas pueden pagar una suma adicional por encima del $7 \%$ obligatorio para obtener una mejor cobertura, en especial en cuanto al pago máximo por atención, y más posibilidades de elección en la atención médica. En 2000, el 50\% de los hogares afiliados a las Isapres aumentaron su cotización para mejorar su cobertura (Superintendencia de Isapres, 2001). Las compañías de seguros del sistema privado están autorizadas para reajustar las cotizaciones y las prestaciones según el riesgo, por razones de sexo, edad y número de cargas familiares (por ejemplo, de acuerdo con un plan común, un soltero de 65 años paga seis veces más que uno de 35). Cada Isapre puede ofrecer cientos de planes diferentes para dar cabida a diversos niveles de ingreso, y en cada uno de ellos difieren sustancialmente el monto del copago y las prestaciones ofrecidas (Larrañaga, 1997). Como las cotizaciones varían bastante según la edad, en 1998 las Isapres dieron cobertura a alrededor de $24 \%$ de los menores de 65 años, pero sólo a 7\% de las personas de 65 años y más (MidePlan, 1999). Aunque casi la totalidad de la atención ambulatoria que se presta a la población afiliada a las Isapres es proporcionada por el sector privado, las personas que cotizan en planes de salud privados suelen recurrir a los establecimientos hospitalarios del sector público. En consecuencia, el uso de estos establecimientos ha significado en la práctica una subvención estatal indirecta al sector privado, ya que el sector público no ha contado con sistemas de información adecuados para cobrarles a las Isapres lo que corresponde.

\section{II}

\section{El papel de la equidad en la atención médica}

En 1990, cuando se restableció la democracia, la "equidad" y el "crecimiento" fueron los pilares de la política pública del Estado chileno (Muñoz Porras, 1998). El crecimiento económico nacional se mantuvo y las tasas de pobreza declinaron durante la mayor parte del decenio de 1990, pero también aumentó la desigualdad económica, medida por el porcentaje del ingreso urbano total percibido por el $10 \%$ de los hogares urbanos más ricos y según el creciente coeficiente de Gini de desigualdad del ingreso (CEPAL, 2001). Con miras a mantener un amplio apoyo popular, el gobierno de centroizquierda se fijó como meta lograr la equidad en los sectores públicamente visibles, como los servicios sociales y de salud, aunque la Constitución de 1980 limitó su acción en esta materia a la estructura política y económica creada por el régimen militar. En el decenio de 1990, las metas del Ministerio de Salud fueron la equidad, la descentralización y la satisfacción/participación de los pacientes (Ministerio de Salud, 1999).

En otros países de América Latina (Alleyne, 2002) y en todo el mundo (oms, 2000; Hurst y Jee-Hughes, 2000), también se ha puesto énfasis en la equidad de 
los servicios de salud, lo que involucra una serie de aspectos diferentes, entre ellos la distribución de los resultados del "proceso" (entendido como la forma en que se entrega la prestación) y del financiamiento. A diferencia del criterio que centra la atención en la eficiencia, en virtud del cual se procura maximizar los indicadores de los resultados y del proceso por insumo financiero, el criterio de equidad examina en qué medida cada uno de estos aspectos se distribuye a través de la población (Hurst, 2001). En Chile son motivo de especial preocupación las diferencias de equidad existentes entre los distintos grupos socioeconómicos y entre las distintas regiones geográficas del país (diferencias que a veces se expresan como las existentes entre zonas urbanas y rurales, pero más a menudo como diferencias entre las 13 regiones administrativas en que se divide de norte a sur el país).

Entre los indicadores de resultados que se consideran importantes en el país, cabe mencionar la mortalidad, en especial la infantil, y la esperanza de vida. En Chile, la primera continúa presentando diferencias según la región y según el grado de instrucción de la madre (Muñoz Porras, 1998; Hollstien y otros, 1998), por mucho que haya disminuido de manera espectacular en los últimos 40 años y que Chile esté disputando el primer lugar a nivel internacional en materia de equidad con respecto a la supervivencia del niño (OMS, 2000; Hurst y Jee-Hughes, 2000). Sin embargo, la mortalidad global sigue acusando desigualdades, puesto que guarda relación con el nivel de ingreso de las comunidades (Arteaga y otros, 2002). En los últimos 40 años ha aumentado la esperanza de vida, incluso entre las personas que han cumplido 60 años, aunque a esa edad la esperanza de vida restante varía entre 21.1 y 23.1 años en las distintas regiones administrativas (Morales y Villalón, 1999).

Los indicadores del proceso describen la forma en que se producen los resultados, incluyendo la calidad de la atención y el acceso a los servicios. Cuando se trata de solicitar atención médica o dental, se observan grandes desigualdades según sexo y nivel de ingreso, lo que indica la existencia de obstáculos al acceso por estos motivos (OPS, 2000; Arteaga y otros, 2002). Además, el grado de satisfacción con el sistema de atención de salud (Rodríguez y Tokman, 2000) está altamente vinculado al ingreso. La Organización Mundial de la Salud (oms) denomina "receptividad" a este elemento de la equidad, y su encuesta entre informantes clave sitúa a Chile en el lugar 45 en cuanto a logros generales en el proceso de salud, pero en el lugar 103 en cuanto a equidad de la receptividad (oms, 2000; Hurst y Jee-Hughes, 2000). La estructura del sis- tema dual público-privado de atención médica y seguro es una de las causas importantes de la desigualdad relacionada con el ingreso que se observa en las prestaciones (Sojo, 1996).

El financiamiento suele medirse por las cotizaciones de cada beneficiario o por lo que se gasta en cada uno de ellos. Las cotizaciones son equitativas cuando comprenden una proporción no regresiva del ingreso de libre disposición de la persona, mientras que el gasto es equitativo si hay una correlación directa entre la necesidad de atención médica y los recursos utilizados. En Chile, el gasto en atención médica no es equitativo, pues el Estado gasta alrededor de 93 mil pesos per cápita al año, mientras que el sector privado gasta 143 mil pesos por persona para atender a una población más sana y más joven (Titelman, 1999). Esta es una estimación mínima de la inequidad del gasto, porque los datos no incluyen los apreciables copagos que se hacen en el sector privado; en la práctica, la diferencia de gasto es bastante mayor. También es motivo de general preocupación la clasificación de riesgo de las Isapres, que lleva a las personas de más edad y mayor riesgo a depender de manera desproporcionada del FONASA, subvencionado por el Estado (Bitrán y Almarza, 2000; Sojo, 1999; Sapelli y Torche, 1998). Contrariamente a su liderazgo mundial en supervivencia del niño, Chile sólo ocupa el lugar 168 entre 191 países en la cuantificación que hace la oms de la equidad de la contribución financiera (OMS, 2000; Hurst y Jee-Hughes, 2000).

El financiamiento de la atención médica en Chile en el decenio de 1990 muestra los niveles más bajos de equidad entre los tres principales aspectos del desempeño de los sistemas de salud: resultados, proceso y financiamiento. En los dos últimos años, las iniciativas de política - como el plan de Acceso Universal con Garantías Explícitas (AUGE) — se han centrado en mejorar el acceso general a las prestaciones médicas de alto costo y a aquéllas con largo tiempo de espera en el sistema público, mediante la aplicación de un sistema de financiamiento más equitativo.

El análisis que figura a continuación contribuye a un mejor conocimiento de los factores que influyen en la equidad del sistema de atención de salud que resulta de estas reformas, mediante el examen de la situación de las personas que más dependen de la atención médica, esto es, las de 65 años o más. La investigación avanza más allá de los estudios efectuados hasta ahora sobre la equidad en los seguros de salud de Chile, que generalmente establecen la dicotomía sector público-sector privado y ofrecen análisis bivariados (por ejemplo, Larrañaga, 1999; Lenz y 
otros, eds., 1999; Muñoz Porras, 1998), pues muestra las variaciones que se observan dentro del sector previsional en su conjunto y efectúa un análisis multivariado del acceso a los servicios médicos.

\section{III}

\section{Metodología}

En el presente estudio se utiliza la Encuesta de Caracterización Socioeconómica Nacional (CASEN) que llevó a cabo el Ministerio de Planificación y Cooperación (MIDEPLAN) en noviembre y diciembre de 1998. En este estudio de alcance nacional se encuestó a un total de 48103 hogares y 188348 personas, 14910 de las cuales tenían 65 años y más. La Comisión Económica para América Latina y el Caribe (CEPAL), organismo regional de las Naciones Unidas, ajustó las cifras de los ingresos declarados para dar cuenta de los datos omitidos e insuficientemente notificados (CEPAL, 1999). Todos los análisis del presente estudio se llevaron a cabo utilizando ponderaciones normalizadas, con el fin de tener en cuenta la desproporción en el tamaño de la muestra de las zonas rurales.

Entre los indicadores del acceso a la atención que utilizó la encuesta figuran los siguientes: si el encuestado sufrió alguna enfermedad o accidente en los últimos tres meses; si en caso de enfermedad recibió atención médica, y si en caso de haberla recibido ésta fue oportuna, con alguna demora o tardía. Se formularon diversas preguntas sobre la atención médica recibida, como el número de veces que el encuestado se había sometido a controles preventivos de salud; si había recibido atención médica ambulatoria y si había consultado a un especialista en los últimos tres meses. Todas estas variables se dividieron en respuestas afirmativas o negativas para cada caso. Si el encuestado había recurrido a los servicios médicos o le habían recetado medicamentos en caso de enfermedad, se le preguntó sobre la forma en que los había pagado. Las respuestas se dividieron en dos clases, una correspondiente a los que no habían gastado dinero de su bolsillo y la segunda a las personas restantes. Finalmente, a las mujeres se les preguntó si alguna vez se habían hecho la prueba de Papanicolau. La pregunta relativa a la cobertura del seguro de enfermedad incluyó una categoría para los que sabían que tenían derecho al plan de seguro estatal pero no tenían certeza acerca de la subcategoría a que pertenecían. Para incluir a estas personas en el análisis de regresión, les imputamos un plan específico de seguro, aplicando un procedimien- to de imputación sobre la marcha (hot-deck) que distinguía entre las personas que vivían en zonas rurales o urbanas, si eran o no casadas, si tenían 70 ó más años de edad o entre 65 y 69 años, si habían cursado o no estudios secundarios, y en qué cuartiles de ingreso familiar per cápita se hallaban. Estos casos representaron el 3\% de las personas afiliadas al sistema previsional estatal. Los demás tipos de asegurados correspondían al sistema de las Fuerzas Armadas, a las Isapres, a "particulares" (entre éstos las personas no afiliadas a Isapres, las cubiertas por planes proporcionados en el empleo y las no aseguradas) y otros.

Entre las características demográficas utilizadas para estratificar la población de usuarios cabe mencionar la edad, el sexo, el nivel de ingresos, el grado de instrucción y la residencia en zonas rurales. Se distinguió entre las personas de 65 a 74 años, y las de 75 años o más. Los ingresos fueron ajustados por la CEPAL de manera de dar cuenta de los valores omitidos e insuficientemente notificados (CEPAL, 1999) y se presentaron como ingreso familiar per cápita. En las regresiones, éste se dividió en dos clases, una correspondiente al quintil más bajo y la otra a los restantes cuatro quintiles de las personas afiliadas a los planes de seguro analizados (el ingreso familiar per cápita del quintil más bajo era de 45 mil pesos mensuales; en comparación, la pensión asistencial de vejez que pagó el Estado en 1998 ascendió a 23 mil pesos mensuales). El grado de instrucción se clasificó según el número de años de escolaridad, y en la regresión se distinguió entre menos que enseñanza secundaria ( 0 a 8 años) y con 1 año o más de educación secundaria. Cabe destacar que en Chile la educación obligatoria no comprende la enseñanza secundaria. Se consideraron como zonas rurales aquellas que se encontraban fuera de las comunidades registradas.

Después de evaluar las distribuciones de frecuencia en las variables socioeconómicas y el acceso a los servicios de salud, se estimaron ecuaciones de regresión logísticas respecto de cada una de las variables de acceso, ajustadas por los indicadores socioeconómicos (educación, ingreso, sexo, edad, residencia en zonas urbanas o rurales). El análisis se limitó a los afiliados 
al seguro estatal general FONASA, al sistema de las Fuerzas Armadas y a las Isapres. Estas clases de seguro se incorporaron como variables categóricas, tomando como grupo de referencia el seguro privado. Puesto que una ecuación de regresión logística única sólo prueba la importancia estadística de las diferencias entre los distintos seguros respecto del grupo de referencia, se resolvieron ecuaciones múltiples cambiando el grupo de referencia, de modo de poder evaluar cada clase de seguro con respecto a todos los demás.

\section{IV}

\section{Resultados del análisis}

Entre las personas de 65 años y más el seguro estatal del FONASA es con mucho el más común (cuadro 1). Una cuarta parte es atendida en el FONASA A (el plan para indigentes), lo que indica que no percibe ni siquiera una pensión o ingreso mínimo; este plan es también el más limitado. Más de un tercio pertenece al FONASA $\mathrm{B}$, lo que indica que su pensión o ingresos se aproximan al mínimo. En su conjunto, el FONASA A y el в cubren al $62 \%$. Los dos niveles con apoyo estatal en que cotizan los adultos mayores con más recursos —esto es, el FONASA C y el FONASA D- abarcan conjuntamente otro $15 \%$. Otro $3 \%$ sabe que tiene derecho al seguro esta- tal, pero no sabe a ciencia cierta en qué nivel. En 1998, el sistema previsional general patrocinado por el Estado abarcaba al $80.4 \%$ de la población de la tercera edad. El 6\% cotizaba en el sistema de las Fuerzas Armadas, y menos de $7 \%$ en las Isapres. Alrededor de $6 \%$ correspondía a personas afiliadas a una serie de otros planes "particulares" —entre ellos programas especiales que cubren incapacidades relacionadas con el trabajo- y a personas que no cotizaban en seguro alguno.

Tanto el promedio como la mediana del ingreso familiar per cápita varían según el tipo de seguro (cuadro 1). Los niveles A, B, C y D del FonASA reflejan en

CUADRO 1

Chile: Características de las personas de 65 años y más según su plan de seguro de salud, $1998^{a}$

\begin{tabular}{|c|c|c|c|c|c|c|c|c|c|}
\hline & $\begin{array}{l}\text { Todo el } \\
\text { país }^{b}\end{array}$ & $\begin{array}{l}\text { FONASA A } \\
\text { (indigentes) }\end{array}$ & FONASA B & FONASA C & FONASA D & 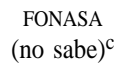 & $\begin{array}{l}\text { Fuerzas } \\
\text { Armadas }\end{array}$ & $\begin{array}{c}\text { Isapres } \\
\text { (privadas) }\end{array}$ & Particular \\
\hline Muestra $\mathrm{n}=$ & $14910^{d}$ & 4817 & 5787 & 738 & 935 & 414 & 520 & 562 & 927 \\
\hline Alcances del plan (\%) & 100 & 25.0 & 37.2 & 6.0 & 9.1 & 3.1 & 5.7 & 6.7 & 5.9 \\
\hline Ingreso promedio & 160223 & 68368 & 107886 & 155048 & 246436 & 213157 & 252555 & 520149 & 222459 \\
\hline Mediana de ingreso ${ }^{\text {ef }}$ & 74784 & 51844 & 74916 & 96185 & 142118 & 104612 & 154155 & 252340 & 98194 \\
\hline $\begin{array}{l}\text { Edad promedio } \\
\text { Personas de } 75 \text { años }\end{array}$ & $73.2 \forall .1$ & $73.7 \forall .1$ & $73.5 \forall .1$ & $72.8 \forall .3$ & $71.8 \forall .2$ & $74.5 \forall .4$ & $73.6 \forall .3$ & $71.0 \forall .3$ & $72.4 \forall .2$ \\
\hline y más $(\%)$ & 35.5 & 39 & 37.2 & 35.4 & 27.7 & 45.9 & 39.9 & 21.8 & 30.3 \\
\hline Mujeres (\%) & 57.3 & 57.4 & 59.9 & 53.4 & 51.1 & 56.9 & 62.2 & 48 & 57.2 \\
\hline $\begin{array}{l}\text { Enseñanza básica } \\
\text { incompleta }(\%)\end{array}$ & 47.8 & 74.3 & 55.1 & 33.8 & 18.6 & 35.2 & 14.4 & 8.5 & 32.9 \\
\hline $\begin{array}{l}\text { Enseñanza secundaria } \\
\text { incompleta }(\%)\end{array}$ & 29.2 & 9.0 & 19.7 & 37.2 & 56.6 & 38.8 & 51.9 & 74.5 & 43.9 \\
\hline $\begin{array}{l}\text { Promedio años de } \\
\text { escolaridad }\end{array}$ & $7.1 \forall .01$ & $3.4 \forall .04$ & $5.0 \forall .05$ & $7.1 \forall .15$ & $9.3 \forall .1$ & $7.0 \forall .22$ & $8.5 \forall .16$ & $11.6 \forall .20$ & $7.5 \forall .15$ \\
\hline $\begin{array}{l}\text { Residencia en zonas } \\
\text { rurales }(\%)\end{array}$ & 17.7 & 36 & 17 & 5.4 & 4.8 & 5.9 & 1.8 & 2.7 & 17.1 \\
\hline
\end{tabular}

Fuente: Elaborado por el autor sobre la base de la investigación.

a FONASA: Fondo Nacional de Salud. Isapres: instituciones de salud previsional. Fuerzas Armadas: sistema de salud de las Fuerzas Armadas.

b Incluye "otros" y "no sabe", que no se indican por separado y representan $1.3 \%$ de los encuestados

c Se refiere a las personas que pertenecen al FONASA pero ignoran en qué nivel están.

d La cifra corresponde a 1085000 habitantes de Chile que tienen 65 años o más.

e El ingreso promedio corresponde al ingreso familiar mensual total per cápita expresado en pesos chilenos, ajustado por la CEPAL de modo de incluir el ingreso insuficientemente declarado.

f Para todo el país, $75^{\circ}$ percentil $=124821$ pesos, $90^{\circ}$ percentil $=238856$ pesos. 
orden ascendente los diferentes niveles de ingreso de los afiliados. La mediana del ingreso familiar per cápita de los con mayores ingresos (FONASA D) duplica la de aquéllos en el grupo de menores ingresos que tienen que cotizar (FONASA B). La de los afiliados al plan de las Fuerzas Armadas es similar a la de los que cotizan en el FONASA D, mientras que la de los afiliados a las Isapres es 1.75 veces superior. La mediana del ingreso familiar per cápita de quienes cotizan en el sistema privado se sitúa levemente por encima del $90^{\circ}$ percentil de los chilenos de la tercera edad. Entre éstos, los afiliados al sistema privado son también más jóvenes, tienen menor probabilidad de ser mujeres y más educación; en otras palabras, lo más común es que sean personas mayores cuyos indicadores demográficos se relacionan con una menor necesidad de asistencia médica y mayores recursos. Las personas con cobertura privada o pertenecientes al sistema de las Fuerzas Armadas son las que registran una menor probabilidad de residir en las zonas rurales. Esto coincide con lo indicado en otros estudios que abarcan todas las edades, los cuales también concluyen que, en su conjunto, las necesidades de la población que se atiende en el sistema público son mayores que las de los afiliados a los planes privados (MIDEPLAN, 2001).

Los indicadores del acceso a los servicios de salud de los adultos mayores según el seguro al que pertenecen también acusan grandes diferencias (cuadro 2). Los afiliados a seguros privados estaban entre aquéllos con menor probabilidad de declarar que habían

CUADRO 2

Chile: indicadores de acceso a los servicios de salud de las personas de 65 años y más según tipo de seguro, 1998a

(Porcentajes)

\begin{tabular}{|c|c|c|c|c|c|c|c|c|c|}
\hline & $\begin{array}{l}\text { Todo el } \\
\text { país }^{b}\end{array}$ & $\begin{array}{l}\text { FONASA A } \\
\text { (indigentes) }\end{array}$ & FONASA B & FONASA C & FONASA D & $\begin{array}{l}\text { FONASA } \\
{\text { (no sabe })^{\mathrm{c}}}^{\text {Fon }}\end{array}$ & $\begin{array}{l}\text { Fuerzas } \\
\text { Armadas }\end{array}$ & $\begin{array}{l}\text { Isapres } \\
\text { (privadas) }\end{array}$ & Particular \\
\hline $\begin{array}{l}\text { Enfermedad/accidente } \\
\text { en últimos tres meses }\end{array}$ & 36.7 & 39.7 & 40.3 & 34.7 & 35.6 & 27.5 & 33 & 28.7 & 27 \\
\hline $\begin{array}{l}\text { En caso de enfermedad/ } \\
\text { accidente, recibió } \\
\text { atención médica }^{\mathrm{d}}\end{array}$ & 82.8 & 77.4 & 83.3 & 85.5 & 93 & 79.8 & 87.9 & 92.3 & 75.4 \\
\hline $\begin{array}{l}\text { Satisfecho con rapidez } \\
\text { con que recibió atención } \\
\text { médica }\end{array}$ & 86.8 & 82.7 & 86.6 & 84.2 & 90.3 & 93.9 & 89.8 & 95.0 & 88.6 \\
\hline $\begin{array}{l}\text { Atención ambulatoria } \\
\text { proporcionada por } \\
\text { proveedor privado }^{\mathrm{d}}\end{array}$ & 24.8 & 7.2 & 27.7 & 36.8 & 48.6 & 52.3 & 17.9 & 91.7 & 61.3 \\
\hline $\begin{array}{l}\text { Atención de urgencia } \\
\text { proporcionada por } \\
\text { proveedor del sector } \\
\text { público }^{\mathrm{e}}\end{array}$ & 82.2 & 88.9 & 86.3 & 85.3 & 89.7 & 69.2 & 89.2 & 32.5 & 50.0 \\
\hline $\begin{array}{l}\text { Consulta médica } \\
\text { preventiva }^{\mathrm{d}}\end{array}$ & 21.4 & 25.3 & 25 & 19.1 & 15.2 & 19.7 & 17.9 & 13.1 & 9.4 \\
\hline $\begin{array}{l}\text { Se sometió alguna vez } \\
\text { a prueba de Papanicolau } \\
\text { (mujeres) }\end{array}$ & 55.8 & 41.7 & 55.1 & 64.0 & 66.4 & 54.7 & 72.1 & 80.8 & 58.4 \\
\hline Consulta de especialista $^{\mathrm{d}}$ & 10.6 & 7.9 & 10.7 & 13.0 & 15.9 & 5.6 & 13.7 & 14.7 & 7.2 \\
\hline $\begin{array}{l}\text { No incurrió en gastos } \\
\text { personales debido a } \\
\text { enfermedad }^{\mathrm{f}} \\
\text { No incurrió en gastos } \\
\text { personales por concepto } \\
\text { de medicamentos }{ }^{\mathrm{f}}\end{array}$ & 58.4 & 89.5 & 65.9 & 39.1 & 25.6 & 34.8 & 5.5 & 6.1 & 15.6 \\
\hline
\end{tabular}

Fuente: Elaborado por el autor sobre la base de la investigación.

a FONASA: Fondo Nacional de Salud; Isapres: instituciones de salud previsional; Fuerzas Armadas: sistema de salud de las Fuerzas Armadas.

b Incluye "otros" y "no sabe", que no figuran por separado y constituyen $1.3 \%$ de los encuestados.

c Se refiere a las personas que pertenecen al FONASA pero ignoran en qué nivel están.

d En los últimos tres meses.

e Comprende el Sistema Nacional de Servicios de Salud (SNSS), el Servicio de Atención Primaria de Urgencia (SAPU) y el sistema de las Fuerzas Armadas.

f Se relaciona con atención ambulatoria recibida. 
estado enfermos o habían sufrido accidentes en los tres meses anteriores, mientras que los pertenecientes al FONASA А у в (que son los grupos de menores ingresos) tenían la mayor probabilidad de hacerlo. Entre quienes declararon haber estado enfermos, los afiliados a las Isapres fueron los que resultaron con mayor probabilidad de haber recibido atención médica, mientras que los pertenecientes al FONASA A y a los planes particulares fueron los que registraron menor probabilidad de haberla recibido (entre éstos, alrededor de la cuarta parte de los que habían declarado enfermedad o accidente no recibieron atención médica). Entre los que sí recibieron atención médica, los afiliados a las Isapres y los cotizantes del sistema de las Fuerzas Armadas acusaron una mayor probabilidad de declarar que habían sido atendidos rápidamente y sin tener que esperar, mientras que las personas con derecho al FONASA A fueron las que menos recibieron atención oportuna. Casi todos los afiliados a los planes de salud privados, así como la mitad de los afiliados al FONASA D, recibieron atención médica ambulatoria de médicos que ejercían libremente la profesión. Las personas con menor probabilidad de haber sido atendidas por esta clase de profesionales fueron las pertenecientes al FONASA A — que recurrieron casi exclusivamente a proveedores públicos - y las que cotizaban en el sistema de las Fuerzas Armadas, la mayoría de las cuales fueron atendidas por profesionales del sistema. Solo 5\% de los adultos mayores declararon haber recurrido a los servicios de urgencia, pero el tipo de atención recibido fue distinto del prestado en otros servicios ambulatorios. Casi $90 \%$ de las personas con cobertura del FONASA o las Fuerzas Armadas que recurrieron a los servicios de urgencia utilizaron proveedores estatales (o de las Fuerzas Armadas), y lo mismo hizo cerca de un tercio de las afiliadas a las Isapres.

El único caso en que las personas de la tercera edad que se atienden en el FONASA A tienen mayor acceso a los servicios de salud corresponde al de quienes declararon haberse sometido a controles preventivos de salud, aunque la tasa absoluta de tales controles $(25 \%)$ es baja para este grupo etario. La tendencia se invierte en las mujeres que declaran haberse hecho un Papanicolau, ya que el $80 \%$ que se da entre las afiliadas a planes de salud privados casi duplica la tasa registrada entre las beneficiarias del FONASA A. Los afiliados al FONASA D, a las Isapres y al sistema de las Fuerzas Armadas fueron los que más consultaron a especialistas, y los que menos consultaron fueron los afiliados al FONASA A y a los planes particulares. Dentro del FONASA se observa, según la mayoría de los indicadores, que el acceso a los servicios de salud aumenta más entre las personas de mayores ingresos (FONASA D) que entre las de menores ingresos (FONASA A).

Entre quienes consultaron médico por motivo de enfermedad o accidente, cerca de $90 \%$ de los pertenecientes al FONASA A declararon no haber incurrido en gastos de su propio bolsillo, frente a $5 \%$ de los que cotizaban en el sistema de las Fuerzas Armadas o en el sistema privado (cuadro 2). Como en el FOnASA A y B el usuario no está obligado a un copago para que lo atiendan en los consultorios del sector público, los que tuvieron que pagar fueron primordialmente los que solicitaron atención privada (7\% de las consultas médicas por enfermedad o accidentes del FONASA A y $28 \%$ de las del FONASA B correspondieron a proveedores privados). En los casos en que se recetaron medicamentos, dos tercios de los afiliados al FonAsA A los recibieron gratuitamente, frente a $5 \%$ de los afiliados a las Isapres y $8 \%$ de los pertenecientes al sistema de las Fuerzas Armadas (cuadro 2).

Aunque podría parecer que el ingreso no debería influir en el acceso a la atención médica de los afiliados al FONASA A (indigentes), los indicadores del acceso de los beneficiarios de este nivel cuyo ingreso familiar per cápita era superior a la mediana fueron significativamente mejores que los de las personas cuyo ingreso era inferior a ésta. Por ejemplo, las personas de la tercera edad con derecho al FONASA A cuyo ingreso familiar per cápita era superior a la mediana registraban una mayor probabilidad que las que se situaban por debajo de la mediana de obtener atención médica en caso de enfermedad $(81.7 \%$ contra $75.9 \%$, $\mathrm{p}<.05)$, y una menor probabilidad de declarar demoras en recibir atención $(9.7 \%$ contra $20.0 \%, \mathrm{p}=.000)$. Como era de prever, entre los adultos mayores afiliados a las Isapres, que deben hacer un copago potencialmente elevado, también se observó un efecto-ingreso, pese a que sus ingresos eran muy superiores a los de las personas pertenecientes al FONASA A. Las personas de edad avanzada afiliadas a las Isapres cuyo ingreso familiar per cápita se situaba en el decil más alto a nivel nacional, acusaban una mayor probabilidad que otros ancianos afiliados a las Isapres (que se situaban principalmente en el octavo y noveno deciles de ingreso) de obtener atención médica en caso de enfermedad $(95.5 \%$ contra $85.5 \%, \mathrm{p}<.05)$ y una menor probabilidad de declarar que habían tenido que esperar para obtener atención médica $(2.1 \%$ contra $8.6 \%, \mathrm{p}=.000)$. Entre las personas de la tercera edad que cotizaban en el sistema de las Fuerzas Armadas se advierte un efecto-ingreso similar. Esta variación, según el ingreso que 
se observa dentro de cada tipo de seguro, indica que al menos parte de las diferencias de acceso entre seguros puede deberse a que el perfil de ingresos de los afiliados a cada uno de ellos es distinto.

Las diferencias entre los tipos de seguro que figuran en el cuadro 3 están ajustadas por algunas de las disparidades de situación socioeconómica, e indican que dentro de ellos se sigue observando grandes discrepancias en el acceso a la atención médica. El cuadro muestra que, comparadas con las personas de la tercera edad afiliadas a las Isapres, las que cotizaban en el sistema de las Fuerzas Armadas tenían posibilidades estadísticamente similares de haber estado enfermas o haber sufrido accidentes en los tres meses anteriores, mientras que las que cotizaban en el FONASA C o D tenían $27 \%$ más posibilidades $(\mathrm{RP}=1.27)$, una vez ajustadas por los indicadores socioeconómicos. En el caso de las personas afiliadas al FONASA A y в, los resultados son similares entre sí, con una mayor probabilidad de $46 \%$ y $47 \%$ de declarar que habían estado enfermas, que las afiliadas a las Isapres. Aunque las razones de posibilidad (RP) que ofrece el cuadro 3 se establecen comparando únicamente con los afiliados a las Isapres, en el cuadro se presentan también comparaciones estadísticas entre los distintos tipos de seguro. Por ejemplo, el riesgo de enfermedad de los afiliados al sistema de las Fuerzas Armadas es estadísticamente similar (=) al de los afiliados a las Isapres y al FONASA C y D, pero la probabilidad de que declaren haber estado enfermos es menor $(<)$ que la de los beneficiarios del FOnASA A о в. Cuando la razón de posibilidad es inferior a 1.0, ello significa que la probabilidad de que el grupo haya notificado el resultado es menor. Por ejemplo, entre las personas que declararon haber estado enfermas o haber sufrido accidentes en los tres meses anteriores, aquéllas con derecho al FONASA A tenían 0.44 veces las posibilidades (esto es, menos de la mitad de las posibilidades, ó $56 \%$ menos) de declarar que habían solicitado atención médica que las afiliadas a las Isapres. Estadísticamente, esta cifra es inferior a la que exhiben los afiliados al FONASA B, al FONASA C y D y a las Isapres, pero no a la de los afiliados al sistema de las Fuerzas Armadas. ${ }^{2}$ Del mismo modo, las personas que habían solicitado atención médica registraron una probabilidad menor de decla-

\footnotetext{
${ }^{2}$ El FONASA A resulta distinto del FONASA c y D, pero no del sistema de las Fuerzas Armadas, principalmente porque el menor tamaño de la muestra de los afiliados a este último exige que las diferencias sean mayores para ser estadísticamente significativas.
}

rar que habían sido atendidas rápidamente si pertenecían al FONASA A, la que aumentaba en el caso del FONASA B, y luego del FONASA C y D, del sistema de las Fuerzas Armadas y de las Isapres en su conjunto. Cuando se agregó una variable adicional por el uso de servicios ambulatorios privados comparado con el de servicios públicos (no indicada), las diferencias entre los distintos seguros en cuanto a la rapidez de la atención disminuyeron al punto de no ser estadísticamente significativas, mientras que la variable de la atención privada resultó significativa $(\mathrm{RP}=2.1, \mathrm{p}=.000)$, lo que indica que el lugar en que se recibió la atención fue más importante que la fuente del seguro.

Al aplicar los controles estadísticos, se advierte que los indicadores globales del acceso a la atención médica de las personas que cotizan en el sistema de las Fuerzas Armadas se aproximan más a los de los beneficiarios de las Isapres y del FONASA c y D, mientras que los indicadores para los afiliados al FONASA A y в suelen parecerse más entre sí que a los correspondientes al FONASA C y D, y son por lo general los peores. En esta matriz, los indicadores de quienes cotizan en el FONASA C y D son siempre distintos de los de quienes están afiliados al FONASA A, mientras que los beneficiarios más acomodados del FonASA C y D se asemejan a los afiliados a las Isapres en dos aspectos, a saber, en lo concerniente a las personas que, habiendo tenido una enfermedad o accidente, declararon haber consultado a un médico, y en lo concerniente a aquéllas que, habiendo tenido una enfermedad o accidente, declararon haber consultado a un especialista. Pese a que las diferencias existentes entre las personas afiliadas a distintos tipos de seguro persisten aun después de ajustar los resultados por las diferencias de situación socioeconómica, es imposible controlar totalmente los resultados por estas diferencias, ya que el FONASA A es, por definición, un seguro asistencial de carácter social que acoge a las personas que carecen de medios para acceder a otro tipo de seguro.

Si bien el cuadro 3 sólo muestra las razones de posibilidad ajustadas según el tipo de seguro, también es útil para describir la evolución de otras variables independientes significativas, a fin de indicar aquellos factores distintos del plan médico que afectan la equidad en el acceso de los adultos mayores a la atención de salud. En las regresiones logísticas implícitamente contenidas en los cuadros, los adultos mayores que vivían en zonas rurales acusaron una mayor probabilidad de declarar que habían estado enfermos, y una menor probabilidad de haber consultado al médico en caso de enfermedad. Asimismo, las personas que residían 
CUADRO 3

Chile: Razones de posibilidad (RP) de acceso a los servicios de salud a través de los seguros públicos ${ }^{\mathrm{a}}$ en comparación con los adultos mayores afiliados a Isapres, ajustadas por sexo, edad, grado de instrucción, ingreso y residencia en zonas rurales ${ }^{b}$

\begin{tabular}{l}
\hline \\
Enfermedad/accidente en últimos 3 meses \\
Diferencias estadísticas respecto de lo anterior, \\
a través de todos los planes
\end{tabular}

a través de todos los planes

En caso de enfermedad, consultaron a un médico Diferencias estadísticas respecto de lo anterior, a través de todos los planes

En caso de haber consultado al médico, satisfechos con rapidez de la atención

Declaran haber consultado a un especialista

Alguna vez se sometieron a prueba de Papanicolau (mujeres)

FONASA A FONASA B FONASA C/D Fuerzas Armadas

$(\text { muestra } \mathrm{n}=1999)^{\mathrm{c}} \quad($ muestra $\mathrm{n}=2407) \quad($ muestra $\mathrm{n}=630) \quad($ muestra $\mathrm{n}=177$ )

\begin{tabular}{|c|c|c|c|}
\hline $\mathrm{RP}=1.46$ & $\mathrm{RP}=1.47$ & $\mathrm{RP}=1.27$ & $\mathrm{RP}=1.12$ \\
\hline$=$ FONASA B & $=$ FONASA A & $<$ FONASA A & $<$ FONASA A \\
\hline$>$ FONASA C/D & $>$ FONASA C/D & $<$ FONASA B & $<$ FONASA B \\
\hline$>$ FF.AA. & $>$ FF.AA. & $=$ FF.AA. & $=$ FONASA C $/ \mathrm{D}$ \\
\hline > Isapres & > Isapres & > Isapres & = Isapres \\
\hline 0.44 & 0.54 & 0.78 & 0.65 \\
\hline$<$ FONASA B & $>$ FONASA A & $>$ FONASA A & $=$ FONASA A \\
\hline$<$ FONASA C/D & $<$ FONASA C/D & $>$ FONASA B & $=$ FONASA B \\
\hline$=$ FF.AA & $=$ FF.AA. & = FF.AA. & $=$ FONASA C $/ \mathrm{D}$ \\
\hline < Isapres & < Isapres & $=$ Isapres & = Isapres \\
\hline
\end{tabular}

\begin{tabular}{|c|c|c|c|}
\hline 0.26 & 0.38 & 0.42 & 0.48 \\
\hline$<$ FONASA B & $>$ FONASA A & $>$ FONASA A & $>$ FONASA A \\
\hline$<$ FONASA C/D & $=$ FONASA C $/ \mathrm{D}$ & $=$ FONASA C $/ \mathrm{D}$ & $=$ FONASA B \\
\hline$<$ FF.AA. & $=$ FF.AA & $=$ FF.AA & $=$ FONASA C/D \\
\hline$<$ Isapres & $<$ Isapres & $<$ Isapres & < Isapres \\
\hline 0.76 & 0.86 & 1.05 & 0.94 \\
\hline$=$ FONASA B & $=$ FONASA A & $>_{S}$ FONASA A & $=$ FONASA A \\
\hline$<$ FONASA C/D & $<$ FONASA C/D & $>$ FONASA B & $=$ FONASA B \\
\hline = FF.AA & $=$ FONASA B & = FF.AA. & $=$ FONASA C/D \\
\hline$<$ Isapres & = Isapres & = Isapres & = Isapres \\
\hline
\end{tabular}

\begin{tabular}{llll}
\multicolumn{1}{c}{1.72} & \multicolumn{1}{c}{1.7} & 1.24 & 1.23 \\
$=$ FONASA B & $=$ FONASA A & $<$ FONASA A & $<$ FONASA A \\
$>$ FONASA C/D & $>$ FONASA C/D & $<$ FONASA B & $<$ FONASA B \\
$>$ FF.AA. & $>$ FF.AA. & $=$ FF.AA. & $=$ FF.AA. \\
$>$ Isapres & $>$ Isapres & $>$ Isapres & $=$ Isapres
\end{tabular}

\begin{tabular}{llll}
$\quad$ & \multicolumn{1}{c}{0.41} & \multicolumn{1}{c}{0.5} & \multicolumn{1}{c}{0.72} \\
< FONASA B & $>$ FONASA A & $>$ FONASA A & $>$ FONASA A \\
< FONASA C/D & $=$ FONASA C/D & $=$ FONASA B & $>$ FONASA B \\
< FF.AA. & < FF.AA. & < FF.AA. & $>$ FONASA C/D \\
< Isapres & $<$ Isapres & $<$ Isapres & $<$ Isapres
\end{tabular}

Fuente: Elaborado por el autor sobre la base de la investigación.

a FONASA: Fondo Nacional de Salud; Isapres: instituciones de salud previsional; FF.AA.: sistema de salud de las Fuerzas Armadas. El FONASA tiene cuatro niveles (A, B, C y D); las personas que dijeron pertenecer al FONASA, pero ignoraban en qué nivel $(\mathrm{n}=414)$, fueron asignadas a uno de los niveles utilizando un procedimiento de imputación sobre la marcha (hot deck).

b Ecuación de regresión logística en que las Isapres son el grupo de referencia $(n=146)$, con control por sexo, edad $(65-69$, 70-74, 75 años y más), grado de instrucción (0-8 años contra 9 y más años), ingreso bajo (<45 000 pesos de ingreso familiar mensual per cápita comparado con cifra superior) y residencia en zonas rurales.

c Los valores $\mathrm{n}$ indican las personas que declararon haber estado enfermas.

en zonas rurales exhibieron una menor probabilidad de declarar que habían consultado a un especialista o se habían sometido a una prueba de Papanicolau (mujeres), pero una probabilidad mayor de declarar que habían sido atendidas oportunamente, con independencia de otras variables. Las mujeres de más edad acu- saron una mayor probabilidad de declarar que habían estado enfermas y también de haber consultado a especialistas o haberse hecho controles preventivos de salud. Por otra parte, los adultos mayores más ancianos mostraron más probabilidades que los adultos mayores de menos edad de declarar que habían estado 
enfermos. Asimismo, entre los de más edad fue mayor la probabilidad de declarar que habían sido atendidos oportunamente y que habían consultado a especialistas, pero menor la de haberse sometido a una prueba de Papanicolau (mujeres). Los ancianos que tenían estudios secundarios acusaron una mayor probabilidad de haber consultado al médico en caso de

\section{$\mathrm{V}$}

\section{Conclusiones}

El aumento de la equidad en la atención de salud se ha convertido en una de las grandes metas del Estado democrático chileno, y de muchos otros países del mundo. Esta meta supone distribuir equitativamente el financiamiento de los servicios de salud, el proceso de atención (incluidos el acceso y la calidad) y los resultados de la atención (oMs, 2000). La mayor parte del debate público acerca de la equidad de los servicios de salud chilenos se ha centrado en las diferencias entre los planes de seguro público y de seguro privado. El presente artículo revela que en el caso del adulto mayor, que es el que más necesita y utiliza los servicios médicos, hay grandes diferencias en el acceso a la atención tanto entre seguros médicos como dentro de ellos. Y puesto que los recursos de que dispone la mayoría de los adultos mayores son limitados, ellos son particularmente vulnerables a la falta de equidad de los servicios médicos.

Sólo los adultos mayores con mejor situación económica pueden hacer frente al costo de los sistemas de seguro privados, debido a que éstos ajustan el riesgo y por lo tanto las cotizaciones de acuerdo con la edad. La investigación mostró que el $7 \%$ de las personas de 65 años y más afiliadas a las Isapres tenían mejor situación económica, mayor grado de instrucción y menos edad que las personas del mismo grupo etario que cotizaban en otros seguros médicos. No es de extrañar que registraran también una menor probabilidad de declarar enfermedad, y que tuvieran asimismo mejor acceso a la atención médica que otros grupos de mayores de 65 años. En otros estudios se ha encontrado que hay personas afiliadas a las Isapres que recurren a los establecimientos hospitalarios estatales, con lo cual el sector público termina subvencionando a quienes tienen seguros privados (MIDEPLAN, 1999; Titelman, 1999) y mejora el acceso a la salud de los enfermedad, de haber consultado a un especialista y de haberse sometido a un Papanicolau (mujeres), pero una probabilidad menor de haberse sometido a controles preventivos de salud. Cualquiera fuese el tipo de seguro, el ingreso se relacionaba con un mayor número de consultas a especialistas y pruebas de Papanicolau (mujeres). afiliados a las Isapres. La presente investigación muestra que en los servicios de urgencia sucede algo similar, ya que un tercio de los adultos mayores afiliados a las Isapres que reciben atención de emergencia recurren a los servicios médicos estatales. Esto sugiere que las personas de la tercera edad afiliadas a seguros privados tienen acceso, según sus necesidades, a los proveedores de servicios médicos tanto públicos como privados. Sin embargo, incluso dentro de este grupo de altos ingresos, las personas con más recursos (esto es, las que pertenecen al decil más alto a nivel nacional) tuvieron mejor acceso a la atención médica que otros adultos mayores afiliados a Isapres. Esto se debe al copago a menudo alto que exigen las Isapres y a la jerarquía de los proveedores de atención privados, entre los cuales los hospitales y clínicas más caros tal vez proporcionen la atención más oportuna y especializada.

El hecho de que las personas de más edad se concentren en el sistema público hace que la equidad dentro de este sistema resulte particularmente importante para este grupo de la población. El principal sistema de seguro de salud del sector público, el FONASA, ofrece distintos tipos de seguro según el monto de las cotizaciones, las que a su vez se basan en el ingreso. Por lo tanto, las características socioeconómicas de los cuatro grupos atendidos por el FONASA son diferentes. Entre la población de la tercera edad, los dos seguros más comunes son el FONASA A, que se destina a los indigentes, y el FONASA B, al que pertenecen las personas con pensiones o ingresos más bajos. Ni uno ni otro requiere copago en el sistema público de atención médica. Sus beneficiarios se asemejan en cuanto a notificación de enfermedad o accidente, consultas médicas en caso de enfermedad, consulta de especialistas y controles preventivos de salud, incluso cuando se toman en cuenta las diferencias en los indicadores 
socioeconómicos (edad, sexo, residencia en zonas rurales, grado de instrucción y bajo ingreso familiar per cápita). En general, los beneficiarios del FONASA C y D (combinados para los efectos del análisis multivariado) tienen mejor situación económica que los del FONASA A O B, pero peor que la de los afiliados a las Isapres. Incluso después de haberse efectuado ajustes por los indicadores socioeconómicos, y pese a los copagos a que están obligados, los afiliados al FONASA c y D tienen mejor acceso a la atención médica que los pertenecientes al FONASA A у в. Las esperas para obtener cita con el médico, que en el sistema estatal han sido un problema crónico durante años, afectaron más que nada a las personas con derecho al FONASA A, no así a las pertenecientes al FONASA B, C y D, ni a los afiliados al sistema de las Fuerzas Armadas. Es posible que la menor demora del FONASA B respecto del FONASA A en la atención del adulto mayor se deba en parte a que los afiliados al FONASA B, pese a sus bajos ingresos, recurrieron con mayor frecuencia a proveedores de salud privados, que pueden servir de válvula de escape cuando hay exceso de demanda en el sistema público. Otros factores que pueden haber contribuido a las diferencias de acceso entre el FONASA A y el B son la insuficiencia de los recursos médicos de la mayoría de las comunidades más pobres, donde probablemente viven los beneficiarios del FONASA A, y también los problemas de gestión que se observan en esas comunidades y que dan lugar a demoras en la prestación de los servicios.

Después del sector privado de seguros, privilegiado desde el punto de vista financiero, entre las personas mayores son los afiliados al seguro de las Fuerzas Armadas, que tienen su propio sistema de atención médica, los que parecen situarse en segundo lugar en cuanto a obtener acceso a atención médica. Le siguen el FONASA B, C y D, que incluyen la opción de recurrir a proveedores privados, y los ancianos que cuentan con mayores ingresos. Las personas de la tercera edad que tienen más necesidades de atención médica y menos recursos se encuentran en el FONASA A, que es el de peor perfil en materia de acceso a los servicios médicos. Como en el sistema estatal estas personas no están obligadas a efectuar copagos, sus problemas de acceso se agravarían mucho más si tuvieran un seguro privado. Al parecer, los ancianos más pobres, que son los del FONASA A, tropiezan además con numerosos obstáculos no relacionados con los planes de salud para obtener la atención médica necesaria, los que no se examinan en el presente estudio. Entre esos obstáculos cabría mencionar el costo del transporte hasta los lugares de atención; problemas de organización de los servicios, como las tan publicitadas colas y esperas en los consultorios públicos, o la insuficiencia de recursos médicos de las comunidades más pobres (Wagstaff, 2001). ${ }^{3}$ La calidad de la atención médica también puede hacer que algunas personas desistan de pedir atención. El $67 \%$ de los encuestados de todas las edades estimó que los proveedores de atención médica estatales eran excelentes, en tanto que el $84 \%$ opinó de igual manera acerca de los proveedores privados. Es importante distinguir entre el asegurador y el proveedor de la atención médica, puesto que aunque el $72 \%$ de los encuestados consideró que el seguro público FONASA era excelente, sólo el $50 \%$ opinó lo mismo de la atención prestada por las aseguradoras privadas, las Isapres (El Mercurio, 2000). El sistema público está tratando de resolver algunos problemas que se interponen en la atención, como las largas listas de espera y la falta de infraestructura adecuada (Gutiérrez, 2000), mientras que el sistema de Isapres procura mejorar su imagen incluyendo la cobertura de enfermedades catastróficas, cuyo tratamiento superaba ampliamente los límites antes establecidos.

El único aspecto en que las personas mayores de bajos ingresos pertenecientes al FONASA A y в obtuvieron mejores resultados que otras fue en la atención preventiva de salud, servicio que todos los planes incluyen y que es gratuito en los consultorios de salud públicos. Sin embargo, las tasas globales son bajas - $13 \%$ para los afiliados a las Isapres y $25 \%$ para los pertenecientes al FONASA A-, lo que indica que la mayoría de las personas más ancianas consideran que la atención médica sólo tiene fines curativos y no preventivos. Es posible que el hecho de que los beneficiarios del FONASA A dependan mucho de los consultorios públicos se traduzca en que sean citados más a menudo para controles preventivos cuando acuden a ellos en caso de enfermedad.

Es importante consignar que para este análisis se dispuso de un número limitado de indicadores de acceso a la atención médica. La mayoría de ellos se refiere a la recepción de distintos tipos de servicios médicos. No se dispuso de información que permitie-

\footnotetext{
3 A manera de ejemplo diremos que un funcionario de gobierno informó acerca de las dificultades con que tropezaban los ancianos enfermos de neumonía para ser hospitalizados en los meses de invierno, cuando la demanda de camas llegaba a su punto máximo. Según dijo, cuando se puso en marcha un programa en virtud del cual los hospitales públicos comenzaron a recibir un aporte adicional por cada adulto mayor enfermo de neumonía hospitalizado, el acceso a la atención mejoró notablemente.
} 
ra tener en cuenta los diversos grados de necesidad de atención médica. Como las personas de bajos ingresos suelen necesitar más servicios médicos que las de mejor situación económica, lo más probable es que si hubiésemos hecho ajustes por concepto de necesidad de atención médica habríamos encontrado un mayor grado de desigualdad en el uso de los servicios. Para identificar maneras eficaces de reducir las desigualdades en la atención médica (independientemente de la redistribución del ingreso), habría que disponer de más datos acerca de los factores que influyen en la recepción de atención médica, como la disponibilidad relativa de servicios, la facilidad con que se puede recurrir a ellos y pagarlos, y la forma en que los distintos grupos evalúan la calidad y la receptividad de la atención recibida.

Debido a que Chile exhibe un satisfactorio crecimiento macroeconómico de largo plazo, a menudo se lo considera un modelo para la formulación de políticas de ajuste estructural. Sin embargo, la legitimidad de los gobiernos democráticos se basa no sólo en los resultados económicos, sino también en los resultados sociales. En Chile, la atención de salud ha sido considerada un sector importante para dar fe de la meta de crecimiento con equidad del gobierno. El presente estudio se centró en la población de 65 años y más porque es la principal usuaria de los servicios de salud y se ve por tanto más afectada por las inequidades del sistema de atención médica. Los datos transversales utilizados no permiten dilucidar si en el decenio de 1990 mejoró la equidad en el acceso a los servicios médicos del adulto mayor, pero efectivamente prueban que en 1998 persistían las desigualdades entre el seguro médico público y el privado, así como también dentro de cada uno de ellos. Gran parte de la controversia sobre la reforma de la salud se ha centrado en las prestaciones y en los mecanismos de financiamiento de los sistemas público y privado. En el presente estudio se muestra que si bien la equidad en las prestaciones y el financiamiento puede ser un componente necesario de la equidad global de los sistemas de salud, no es sin embargo condición suficiente, puesto que hay diferencias de acceso dentro de los diversos tipos de seguros. Esto es particularmente significativo en el caso de los adultos mayores, pues una cuarta parte de ellos pertenece al FONASA A, que brinda el nivel más bajo de acceso a los servicios de salud.

En el actual sistema público de salud, las personas que tienen recursos financieros moderados pueden pagar una suma adicional para ser atendidas en el sistema médico privado, pertenezcan o no a una Isapre. Sin embargo, los proveedores de atención médica privados se encuentran principalmente en los barrios de mayores ingresos: los ancianos de bajos ingresos difícilmente podrían recurrir a este medio, aunque fuera gratuito y estuviera abierto a todos los afiliados al sistema público. En consecuencia, para elevar el acceso a atención médica de las personas mayores (elemento de equidad del proceso), lo más eficaz y viable sería seguir mejorando el funcionamiento de los prestadores del sector público, probablemente aumentando los pagos que deben hacerles las Isapres cuando sus afiliados recurran a los servicios de urgencia y establecimientos hospitalarios públicos, o bien asignando más recursos públicos. También habría que continuar mejorando la organización y gestión de los servicios médicos del sector público (Sojo, 1999). Es fundamental que, cuando se discutan políticas de reforma de los sistemas de salud en Chile y en cualquier otro país que emprenda la modificación de su sistema de financiamiento o suministro de servicios de salud, junto con la equidad en el financiamiento se tenga en cuenta la necesidad de dar al adulto mayor acceso equitativo a los servicios médicos.

Bibliografía

Alleyne, G. A. (2002): La equidad y la meta de salud para todos, Revista panamericana de salud pública, vol. 11, $\mathrm{N}^{\circ} 5-6$, Washington, D.C., Organización Panamericana de la Salud (OPS).

Arenas de Mesa, A. (2000): El sistema de pensiones en Chile: resultados y desafíos pendientes, serie Seminarios y conferencias, $N^{\circ}$ 2, LC/L.1399-P, Santiago de Chile, Comisión Económica para América Latina y el Caribe (CEPAL)/Centro Latinoamericano y Caribeño de Demografía (CELADE).

Arteaga, O. y otros (2002): Información para la equidad en salud en Chile, Revista panamericana de salud pública, vol. 11, $\mathrm{N}^{\circ}$ 5-6, Washington, D.C., Organización Panamericana de la Salud (OPS).
Banco Mundial (1995): Chile: The Adult Health Policy Challenge, Washington, D.C.

Bitrán, R. y F. Almarza (2000): Las instituciones de salud previsional (ISAPRES) en Chile, en D. Titelman y A Uthoff (comps.), Ensayos sobre el financiamiento de la seguridad social en salud: los casos de Estados Unidos, Canadá, Argentina, Chile y Colombia, vol. 2, Santiago de Chile, Fondo de Cultura Económica (FCE)/CEPAL.

Bitrán, R. y otros (1996): Estudio sobre la equidad en el financiamiento del seguro público de salud, Santiago de Chile, Bitrán y Asociados.

Borzutsky, S. (1999): Chile: The politics of privatization, en M. A. Cruz y otros (eds.), Do Options Exist? The Reform of Pension 
and Health Care Systems in Latin America, Pittsburgh, University of Pittsburgh

CEPAL (Comisión Económica para América Latina y el Caribe) (1999): Chile, Encuesta de Caracterización Socioeconómica Nacional, CASEN, 1998, Manual del usuario, intranet de la CEPAL-BADEHOG.

(2001): Panorama social de América Latina 2000-2001, LC/G.2138-P, Santiago de Chile.

Chile, Ministerio de Salud (1999): Situación de la salud en Chile: Tarjeta de presentación, Santiago de Chile, Ministerio de Salud.

El Mercurio (2000): Mala evaluación de las Isapres, microbuses y las municipalidades, A1, Santiago de Chile, 13 de octubre.

Esping-Andersen, G. (1994): After the Golden Age: The Future of the Welfare State in the New Global Order, Occasional paper, $\mathrm{N}^{\circ} 7$, Ginebra, Instituto de Investigaciones de las Naciones Unidas para el Desarrollo Social.

Gutiérrez, O. (2000): Plan piloto en salud reduce lista de espera, $E l$ Mercurio, C4, Santiago de Chile, 25 de diciembre.

Hollstien, R. y otros (1998): Socioeconomic level and infant mortality in Chile in the period 1985-1995, La revista médica de Chile, vol. 126, $\mathrm{N}^{\circ} 3$, Santiago de Chile, Sociedad Médica de Santiago.

Hurst, J. (2001): Performance measurement and improvement in OECD Health systems: overview of issues and challenges, en OCDE (Organización de Cooperación y Desarrollo Económi$\cos )$, OECD Health Conference on Performance Measurement and Reporting, París.

Hurst, J. y M. Jee-Hughes (2000): The performance measurement and performance management in OECD health systems, Occasional papers, $N^{\circ} 47$, Dirección de Educación, Empleo, Trabajo y Asuntos Sociales, París, Organización de Cooperación y Desarrollo Económicos (OCDE).

Larrañaga, O. (1997): Eficiencia y equidad en el sistema de salud chileno, serie Financiamiento del desarrollo, $\mathrm{N}^{\circ}$ 49, LC/L.1030, Santiago de Chile, CEPAL.

(1999): Health sector reforms in Chile, en D. Leipziger y G. Perry, Chile: Recent Policy Lessons and Emerging Challenges, Washington, D.C., Banco Mundial.

Lenz A. y otros (eds.) (1999): FONASA: ¿una reforma en marcha?, Santiago de Chile, Fondo Nacional de Salud (FONASA).

MIDEPLAN (Ministerio de Planificación y Cooperación) (1999): Situación de la salud en Chile, 1998, Santiago de Chile, División Social, Departamento de Estudios Sociales.

(2001): Situación de la salud en Chile 2000, Documento $\mathrm{N}^{\circ}$ 5, Santiago de Chile, División Social, Departamento de Estudios Sociales.
Ministerio de Salud (1999): Situación de la salud en Chile: tarjeta de presentación, Santiago de Chile.

Morales, I. y J. Villalón (1999): Chile y los adultos mayores. Impacto en la sociedad del 2000, Santiago de Chile, Presidencia de la República, Comité Nacional para el Adulto Mayor/ Instituto Nacional de Estadísticas (INE).

Morley, S., R. Machado y S. Pettinato (1999): Indexes of Structural Reform in Latin America, serie Reformas económicas, $\mathrm{N}^{\circ} 12$, LC/L.1166, Santiago de Chile, CEPAL.

Muñoz Porras, F. (1998): Equidad y eficiencia en la toma de decisiones del sistema de salud chileno, en H. Salinas, Equidad y eficiencia en la toma de decisiones en salud, Santiago de Chile, Ministerio de Salud.

OMS (Organización Mundial de la Salud) (2000): World Health Report 2000-Health systems: Improving Performance, Ginebra.

OPS (Organización Panamericana de la Salud) (1998): Health in the Americas, Washington, D.C.

(2000): Hojas-Resumen sobre desigualdades en salud: Chile, Washington, D.C., División de Salud y Desarrollo Humano.

Rodríguez, J. y M. Tokman (2000): Resultados y rendimiento del gasto en el sector público de salud en Chile 1990-1999, serie Financiamiento del desarrollo, $\mathrm{N}^{\circ}$ 106, LC/L.1458-P, Santiago de Chile, CEPAL

Sapelli, C. y A. Torche (1998): El seguro previsional de salud: determinantes de la elección entre el seguro público y privado, 1990-1994, Cuadernos de economía, vol. 35, № 106, Santiago de Chile, Pontificia Universidad Católica de Chile.

Sojo, A. (1996): Reformas de gestión en la salud pública en Chile, serie Políticas sociales, $\mathrm{N}^{\circ} 13$, LC/L.933, Santiago de Chile, CEPAL.

(1999): Los compromisos de gestión en salud de Chile: formas de reintegración en el rompecabezas de la reforma, A. Lenz y otros (eds.) FONASA: ¿una reforma en marcha?, Santiago de Chile, FONASA.

Superintendencia de Isapres (2001): Balance de gestión 2000 y desafios 2001, Santiago sde Chile.

Titelman, D. (1999): Reformas al financiamiento del sistema de salud en Chile, Revista de la CEPAL, N 69, LC/G.2067-P, Santiago de Chile, CEPAL.

Villa, M. y L. Rivadeneira (2000): El proceso de envejecimiento de la población de América Latina y el Caribe: una expresión de la transición demográfica, serie Seminarios y conferencias, $\mathrm{N}^{\circ}$ 2, LC/L.1399-P, Santiago de Chile, CEPAL/CELADES.

Wagstaff, A. (2001): Poverty, equity, and health: Some research findings, en OPS, Equity and Health: Views from the Pan American Sanitary Bureau, Washington, D.C. 